\title{
Nonlinear low noise particle-in-cell simulations of electron temperature gradient driven turbulence
}

\author{
A. Bottino and A. G. Peeters \\ Max-Planck-Institut für Plasmaphysik, IPP-EURATOM Association, Garching, Germany \\ R. Hatzky \\ Computer Center of the IPP and Max-Planck-Gesellschaft, Garching, Germany
}

S. Jolliet, B. F. McMillan, T. M. Tran, and L. Villard

Centre de Recherches en Physique des Plasmas, EPFL, Lausanne, Switzerland

(Received 10 October 2006; accepted 6 December 2006; published online 5 January 2007)

\begin{abstract}
In this Letter, it is shown that global, nonlinear, particle-in-cell (PIC) simulations of electron temperature driven turbulence recover the same level of transport as flux-tube codes when the level of statistical noise, associated with the PIC discretization, is sufficiently small. An efficient measure of the signal-to-noise ratio, applicable to every PIC code, is introduced. This diagnostic provides a direct measure of the quality of PIC simulations and allows for the validation of analytical estimates of the numerical noise. Global simulations for values of $\rho_{e}^{*}<1 / 450$ (normalized electron gyroradius) show no evidence of a gyro-Bohm scaling. (C) 2007 American Institute of Physics. [DOI: $10.1063 / 1.2428280]$
\end{abstract}

Electron temperature gradient (ETG) driven turbulence may be responsible for the high level of residual electron transport observed in many tokamak internal transport barrier experiments. The linear physics of ETG modes is well known and is similar to that of ion temperature gradient (ITG) modes, with the roles of ions and electrons reversed. ${ }^{1}$ Therefore, estimates of ETG induced transport based on mixing length arguments predict diffusion coefficients that are $\sqrt{m_{e}} / m_{i}$ smaller than in the ITG case. However, ETG and ITG turbulence have very different nonlinear behaviors due to the zonal flow response to the turbulence. ${ }^{2,3}$ This is associated with the different origin of the Boltzmann (adiabatic) response of ions (ETG) and electrons (ITG). In the ETG case, the ion adiabatic response arises from the gyro motion due to the large ion gyroradius and it is finite for all flow components. On the other hand, in ITG turbulence the electron Boltzmann response is due to the fast parallel electron motion, whose flow component vanishes. Quantitative ETG transport predictions made with flux-tube simulations ${ }^{2,4}$ showed that, for certain range of physical parameters, the nonlinear turbulent heat flux can be significantly larger compared to the equivalent ITG case, leading to experimentally relevant levels of radial heat transport $\left(\chi_{e} \simeq 13 \chi_{\mathrm{gB}}\right)$. Such high transport levels are attributed to the presence of radially elongated turbulence structures (streamers) and to the consequent $E \times B$ convection. On the other hand, recent global particle-in-cell (PIC) simulations ${ }^{5,6}$ yielded a lower level of anomalous transport than local flux-tube Eulerian codes. ${ }^{2}$ Radially elongated streamers with widths of several ion Larmor radii have been identified, but their impact on the transport was found to be small. Although the streamer size scales with the device size, the transport scaling was found to be gyro-Bohm (gB). Two possible explanations have been proposed to account for this discrepancy: a new nonlinear saturation mechanism associated with toroidal mode coupling ${ }^{5}$ and the influence of the nonphysical statistical noise due to the particle discretization. ${ }^{7}$ The latter paper shows that in PIC simulations, a large level of statistical noise can reduce the ETG turbulence induced transport and can determine the saturation level of the instability.

The purpose of this Letter is to clarify the discrepancy between flux-tube and global PIC results by analyzing a set of nonlinear global PIC simulations in which the level of statistical noise is measured and controlled. Results show that low noise PIC simulations produce levels of transport comparable with flux-tube simulations even for relatively large values of $\rho_{e}^{*} \equiv \rho_{v_{\text {th }, e}} / a$. Here, $\rho_{v_{\text {th }, e}}=v_{\text {th }, e} / \Omega_{e}$, where $v_{\text {th }, e}$ is the electron thermal velocity and $\Omega_{e}$ is the electron cyclotron frequency. The statistical noise clearly does have a strong influence on the nonlinear behavior of ETG turbulence, in agreement with the results of Ref. 7. In this Letter, we also introduce a measure of the statistical noise based on a signal-to-noise ratio, which provides a direct and trustworthy indicator of the quality of the PIC simulations. We also show that this diagnostic allows for the validation of analytical estimates for the statistical noise. All the simulations presented in this Letter have been performed with the global gyrokinetic electrostatic code ORB $5,{ }^{8-10}$ which uses several noise reduction techniques, including optimized particle loading, ${ }^{12}$ Fourier filtering, and an adaptive gyro-averaging procedure. The code ORB5 solves the gyrokinetic Vlasov equation ${ }^{13}$ using marker particles. The projection of the markers on a fixed grid (charge assignment) provides the source term for the quasineutrality equation, which is discretized using finite elements (B-splines). Basic physical conservation properties, i.e., energy and particle number, are used as indicators of the quality of the numerical simulations. In all the ETG simulations presented in this work, ions are assumed to be adiabatic. Equilibrium $n$ and $T_{e}$ profiles self-consistently evolve in time; i.e., sources are not included 


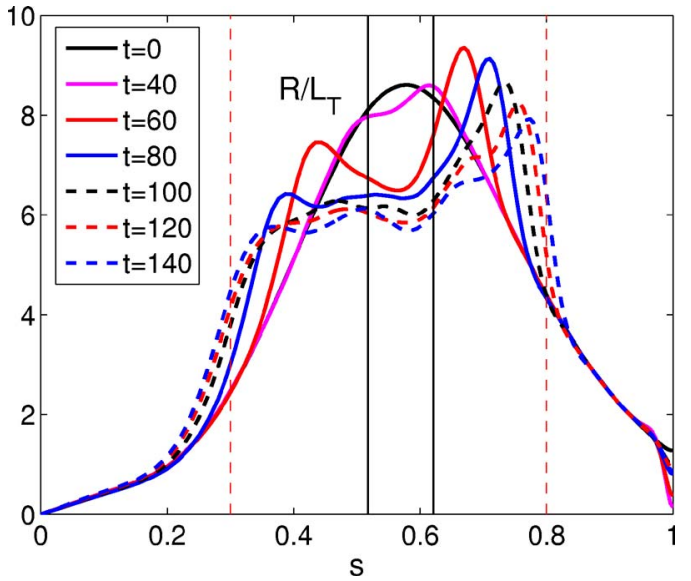

FIG. 1. (Color online) Evolution of the electron temperature gradient in time $\left[a / v_{T_{e}}\right]$. CYCLONE base case, $\rho_{e}^{*}=1 / 450,512 \times 10^{6}$ markers.

in the simulations. The simulation parameters are basically the CYCLONE $^{14}$ case nominal values for the aspect ratio, safety factor, and density profile $\left[R / L_{n}(r / a \equiv 0.5)=2.2\right]$, while $\rho_{e}^{*}=1 / 450,1 / 320$, and $1 / 160$.

Figure 1 shows the temperature gradient evolution $\left(R / L_{T}\right)$ for a $\rho_{e}^{*}=1 / 450$ circular plasma simulated with 512 $\times 10^{6}$ marker particles. The value of $R / L_{T}$ decreases in time in the region of maximum gradient and increases radially toward plasma edge and center (radial spreading). Time is measured in $\left[a / v_{\text {th }, e}\right]$ units, and the radial variable is $s$ $\equiv \sqrt{\psi_{\text {pol }}}$. The vertical dashed lines in Fig. 1 enclose the radial region in which the temperature gradient exceeds the critical value of the ETG mode (in this case, $R / L_{T, \text { crit }} \simeq 4$.6) at the end of the simulation. The formation of radially elongated streamers is observed. The radial length of the streamers continuously increases in time following the radial spreading of the temperature gradient and does not appear to converge to a saturation value. Streamers cover the entire radial region where drive is present; i.e., $R / L_{T}>R / L_{T \text {,crit. }}$ This is illustrated in Fig. 2, where the dashed flux surfaces correspond to the vertical dashed line in Fig. 1: radially elongated streamers in the electrostatic potential fill the entire radial cross section where the ETG mode is still unstable, reaching lengths of several $\rho_{i}^{*}$. The same behavior has been observed for all the different values of $\rho_{e}^{*}$ used in this work. Therefore, the radial extent of the streamers depends on the choice of the initial temperature gradient profile. As will be discussed later, the radial spreading of the streamers occurs together with a nonlinear downshift of the poloidal wave number spectrum. This suggests that, in addition to the linear drive arising from the temperature gradient spreading, turbulence spreading associated with nonlinear toroidal mode coupling could also play a role in determining the radial evolution of the streamer size. However, these two effect cannot be easily decoupled in freely evolving profile simulations.

The time evolution of the average value of $R / L_{T}$ and of the diffusion coefficient $\chi / \chi_{\mathrm{gB}}$ (radial average over $0.52<s$ $<0.62$, black vertical lines in Fig. 1) is plotted in black in Fig. 3. During the overshoot, the temperature gradient strongly relaxes and falls below the nominal value of the CYCLONE base case $R / L_{T}=6.9$. For $t>80, R / L_{T}$ decays

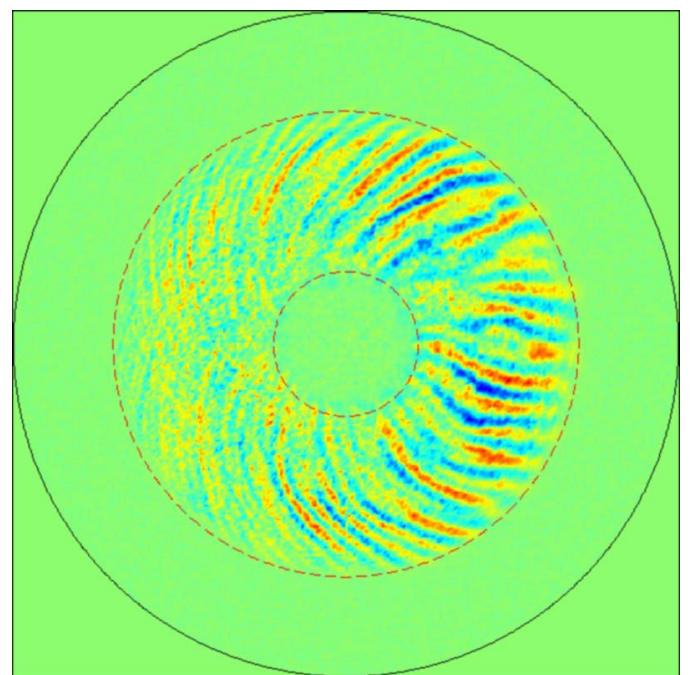

FIG. 2. (Color online) Cross section of the electrostatic potential at $t=140$ $\left[a / v_{T_{e}}\right]$. CYCLONE base case, $\rho_{e}^{*}=1 / 450,512 \times 10^{6}$ markers.

slowly from $R / L_{T} \simeq 6.5\left(\chi / \chi_{\mathrm{gB}} \simeq 17\right)$ to $R / L_{T} \simeq 5.8\left(\chi / \chi_{\mathrm{gB}}\right.$ $\simeq 8.5$ ). Taking into account that the overshoot depends strongly on the arbitrary initial perturbation, the slowly decaying phase is the only physically relevant part of the simulation. A possible extrapolation for $R / L_{T} \simeq 6.9$, considering a linear fit of $\chi\left(R / L_{T}\right)$ on the slowly decaying phase only (linear fit for $t>100)$ gives $\chi /\left(v_{v_{\mathrm{th}, e}} \rho_{T e}^{2} / L_{T}\right) \simeq[10,15]$, in agreement with the results of flux-tube simulations (more details can be found in Ref. 15). It is important to notice that in the range $160<\rho_{e}^{*-1}<450$, the scaling of the transport is not gyro-Bohm, as is illustrated in Fig. 3. Therefore, for more realistic values of $\rho_{e}^{*}$, the transport induced by ETG turbulence may be even larger than the estimated value for the $\rho_{e}^{*}=1 / 450$ simulations. Note that in Ref. 5, where a gyroBohm scaling of ETG transport was identified, smaller values of $\rho_{e}^{*}\left(500<\rho_{e}^{*-1}<2000\right)$ have been used.

In numerical modeling, and PIC simulations in particular, careful attention must be paid to convergence issues. PIC codes are subject to statistical noise due to the use of marker particles to sample the phase space. The "statistical noise" is

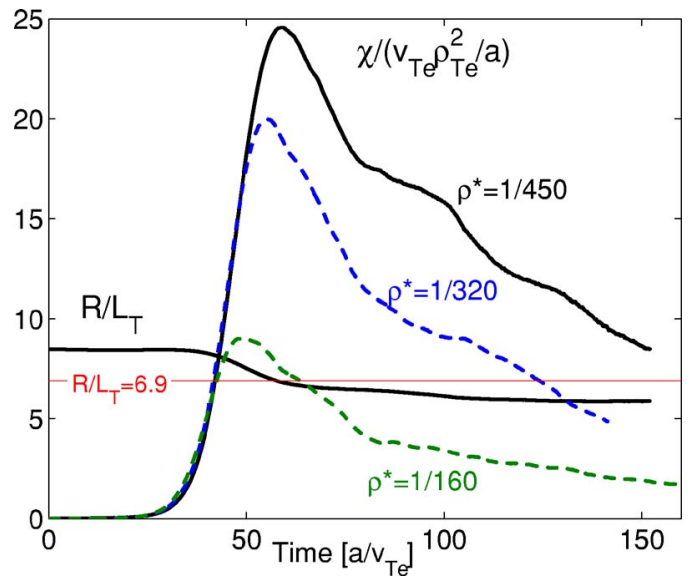

FIG. 3. (Color online) Time evolution of radial averaged $R / L_{T}$ for $\rho_{e}^{*}$ $=1 / 450$ and $\chi / \chi_{\mathrm{gB}}$ for $\rho_{e}^{*}=1 / 450,1 / 320,1 / 160$. Parameters are taken from the CYCLONE base case. The radial average is over $0.52<s<0.62$. 


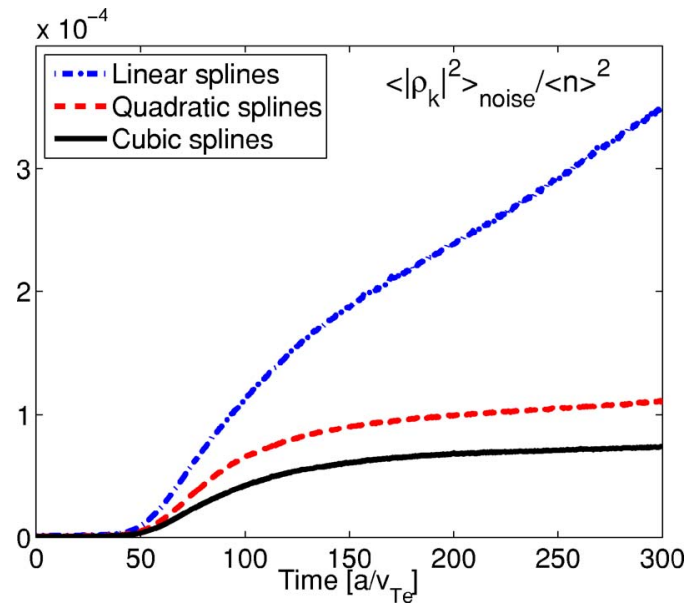

FIG. 4. (Color online) Scaling of the numerical noise in the charge density with the order of B-splines used in the ORB5 simulations. CYCLONE base case, $\rho_{e}^{*}=1 / 80 ; 64$ markers per Fourier mode.

related to the error introduced when moments of the distribution function (for example, the charge assignment) are computed using a finite number of tracers in phase space. ${ }^{16}$ The Monte Carlo theory allows an estimate for the contribution of the noise to the charge density $\rho$ :

$$
\rho_{\text {noise }}^{2} \simeq \frac{N_{G}}{N_{T}}\left\langle w^{2}\right\rangle G, \quad\left\langle w^{2}\right\rangle \equiv \frac{1}{N_{T}} \sum_{i=1}^{N_{T}} w_{i}^{2},
$$

where $N_{T}$ is the number of tracers, $N_{G}$ is the number of modes kept in the simulation, and $w_{i}$ is the weight of a single marker. Details about the derivation and validation of Eq. (1) will be given elsewhere (see also Ref. 15). The function $G$ accounts for additional filtering coming through finite Larmor radius effects and the grid projection algorithm. Equation (1) indicates that the statistical noise can be reduced either by increasing the number of tracers $\left(\sqrt{N_{T}}\right.$ convergence) or by reducing the number of Fourier modes kept in the simulations (Fourier filtering of nonresonant modes). It is important to notice that in standard $\delta f$ PIC simulations, the quantity $\left\langle w^{2}\right\rangle$, and consequently, $\rho_{\text {noise }}^{2}$, grow monotonically in time. ${ }^{11}$ Therefore, an important role is played by the control of the evolution of the variance of the distribution of the weights $\sigma \propto\left\langle w_{i}^{2}\right\rangle$ (optimized loading). ${ }^{12}$ A new diagnostic has been implemented in the code that allows for a direct evaluation of $\rho_{\text {noise }}$. This measure is based on the average amplitude of the contribution to the charge density, i.e., $\left|\rho_{k}\right|$, of the nonresonant (filtered) modes that are physically damped and whose amplitude arises merely from noise. Through the comparison with the charge density of the "physical" modes, a signal-to-noise ratio can be constructed. This diagnostic provides a direct indicator of the quality of the numerical simulations during all the time evolution. Moreover, it can be used for validating analytical estimates of the statistical noise such as the one proposed in Ref. 7 or the simple estimate of Eq. (1). Several ORB5 simulations showed that the scaling of the noise in the number of particles per mode, i.e., $N_{T} / N_{G}$, is in excellent agreement with the estimate of Eq. (1). ${ }^{15}$ Moreover, the scaling of the noise with the number of particles shows that the important parameter in PIC simulations is

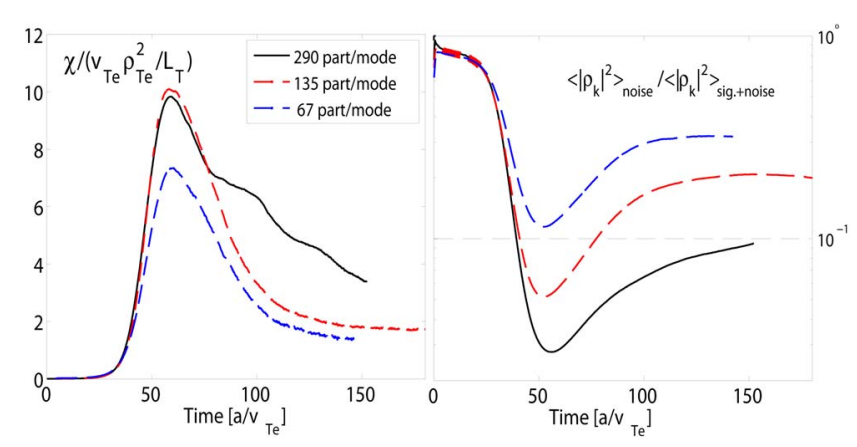

FIG. 5. (Color online) Left: Time evolution of $\chi / \chi_{\mathrm{gB}}$ for different number of markers per mode $\left(N_{T} / N_{G}\right)$. CYCLONE base case, $\rho_{e}^{*}=1 / 450$. Right: Noiseto-signal ratio as function of time for these simulations. CYCLONE base case, $\rho_{e}^{*}=1 / 450$.

indeed the number of particles per Fourier mode and not the number of particles per grid cell. A detailed analysis of the scaling of the statistical noise and its impact on physical quantities has been performed and it will be discussed elsewhere. However, it is important to stress the role of the $G$ function: Although the number of numerical particles per mode is a universal scaling for the noise in PIC codes, the scaling factor, i.e., the $G$ function, is strongly algorithm dependent, and therefore code dependent. For example, different projection algorithms in the charge assignment procedure can lead to very different level of noise: Fig. 4 shows that in ORB5, the level of noise is strongly reduced when moving from linear to cubic finite elements. A more detailed analysis shows that linear B-splines require a factor of 4 in the number of particles (for the same number of grid points) as compare to cubic B-splines to obtain the same noise properties. Moreover, Fig. 4 shows that the statistical noise always grows in time following the time evolution of $\left\langle w^{2}\right\rangle$.

The effect of the statistical noise on ETG simulations is illustrated in Fig. 5 (left). The statistical noise reduces the level of transport driven by ETG turbulence. Figure 5 (right) shows the noise-to-signal ratio for these simulations. These simulations were performed with a "white noise" initial perturbation on all the Fourier modes. Therefore, at the begin-

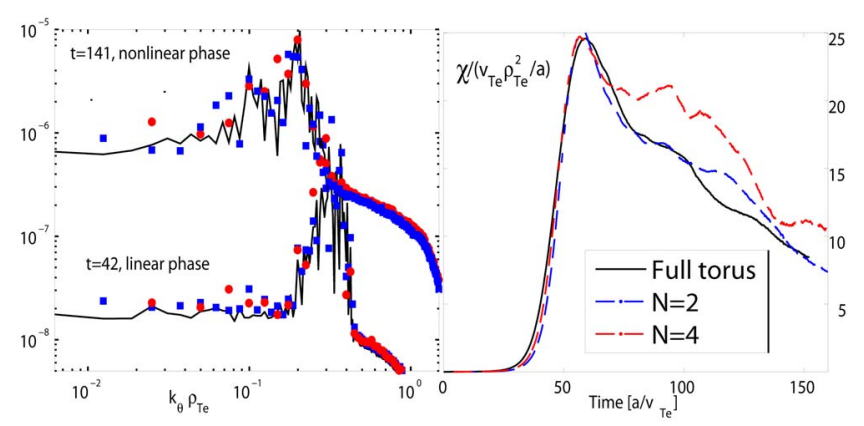

FIG. 6. (Color online) Left: $k_{\theta} \rho_{T_{e}}$ spectrum or full torus, half-torus $(N=2)$, and quarter-torus $(N=4)$ flux-tube filtered simulations. CYCLONE base case, $\rho_{e}^{*}=1 / 450$, number of markers per Fourier mode $N_{T} / N_{G} \simeq 290$. Right: Time evolution of $\chi / \chi_{\mathrm{gB}}$ full torus, half-torus $(N=2)$ and quarter-torus $(N$ =4) flux-tube filtered simulations. CYCLONE base case, $\rho_{e}^{*}=1 / 450$, number of markers per Fourier mode $N_{T} / N_{G} \simeq 290$. 
ning of the simulation, where the signal is close to zero, the signal-to-noise ratio is expected to be around unity, as it is clearly recovered by our diagnostics [Fig. 5 (right)]. In ORB5, single or multimode initialization are also possible. We have performed several simulations in which linearly unstable modes only were initialized, leading to a signal-to-noise ratio of the order of few percent at the early stage of the simulation and to a level of noise in the nonlinear phase that is comparable to the white noise initialization cases. However, single mode initialization usually leads to larger overshoots and consequently to larger profile relaxation before the slowly decaying phase. Figure 5 shows that the results start to diverge when the statistical noise becomes larger than $10 \%$ of the signal. Note that the slow decaying phase (the only physically relevant phase) disappears and it is replaced by a saturated state at very low values of $\chi / \chi_{\mathrm{gB}}$. This result confirms the predictions of Ref. 7. When fewer than 100 particles per mode are used, the noise-to-signal ratio is higher than $10 \%$ during the entire simulation. In this case, even the linear growth rate is not correctly computed. Several tests performed on larger $\rho_{e}^{*}$ cases showed in general that a signal-to-noise ratio $<10 \%$ is required to get converged results, which corresponds to an average value of around 200 markers per mode.

In addition to the reduction of linear drive proposed in Ref. 7, we have observed that the statistical noise creates spurious zonal flows that could contribute to the stabilization of the turbulence.

We have investigated the role of the nonlinear toroidal coupling of the modes in determining the nonlinear saturation of the turbulence. We have performed a set of flux-tube filtered simulations in which only a subset of toroidal modes $(n=0, \pm N, \pm 2 N, \ldots)$ are kept in the Poisson equation. These simulations were performed with the same number of markers per mode and show comparable levels of statistical noise. The time evolution of the poloidal wave number spectrum is shown in Fig. 6 (left). The spectrum of the full torus simulation (solid lines) is consistent with the PIC simulations of Ref. 5. In particular, we observe a nonlinear downshift of the most unstable modes from $0.2<k_{\theta} \rho_{T_{e}}<0.4$ during the linear phase to $0.1<k_{\theta} \rho_{T_{e}}<0.3$ at the end of the simulation. However, flux-tube filtered simulations keeping fixed $N_{G} / N_{T}$ do not show significant differences in the downshift of the $k_{\theta} \rho_{T_{e}}$ spectrum [Fig. 6 (left)]. The level of transport is slightly higher for a quarter-torus simulation $(N=4)$ as compared to full torus simulations as illustrated in Fig. 6 (right). In conclusion, flux-tube filtered results show an increase of the level of transport as compared to full torus, which is consistent with the predictions of Ref. 5. However, this effect appears to be small since half-torus simulations indeed reproduce the correct level of radial transport for $\rho_{e}^{*}=1 / 450$.
In summary, global PIC simulations of freely decaying ETG turbulence for the CYCLONE base case $\left(\rho_{e}^{*}=1 / 450\right)$ show levels of transport comparable with flux-tube simulations when the statistical noise is sufficiently low. GyroBohm scaling is not observed for $\rho_{e}^{*} \leq 1 / 450$. In global PIC simulations, statistical noise reduces ETG driven transport and the level of noise scales with the number of particles per mode, but the scaling coefficient strongly depends on the algorithms used in each code. Radially elongated streamers scale with machine size and cover the whole extend of the drive domain. Flux-tube filtered simulations, preserving the double periodicity, show a similar spectrum (nonlinear downshift) but (slightly) higher levels of transport than full torus simulations. This result is consistent with the existence of a saturation mechanism based on the nonlinear toroidal mode coupling. However, for the parameters used in this work, computationally costly full torus simulations are not needed and flux-tube filtered simulations (for relatively small values of $N$ ) can correctly predict the level of radial transport induced by ETG turbulence.

\section{ACKNOWLEDGMENTS}

Simulations were performed on the IBM pSeries Supercomputer "Regatta" of the MPG-IPP Rechenzentrum, Garching, and on the cluster PLEIADES2 of the EPFL, Lausanne.

${ }^{1}$ F. Jenko, Comput. Phys. Commun. 125, 196 (2000).

${ }^{2}$ F. Jenko and W. Dorland, Phys. Rev. Lett. 89, 225001 (2002).

${ }^{3}$ B. D. Scott, Phys. Plasmas 12, 102307 (2005).

${ }^{4}$ W. Dorland, F. Jenko, M. Kotschenreuther, and B. Rogers, Phys. Rev. Lett. 85, 5579 (2000).

${ }^{5}$ Z. Lin, L. Chen, and F. Zonca, Phys. Plasmas 12, 056125 (2005).

${ }^{6}$ Z. Lin, L. Chen, Y. Nishimura et al., Proc. 20th IAEA Fusion Energy Conference 2004, Vilamoura, Portugal, 2004 (IAEA, Vienna, 2005), Paper TH8-4.

${ }^{7}$ W. M. Nevins, G. W. Hammett, A. M. Dimits et al., Phys. Plasmas 12, 122305 (2005).

${ }^{8}$ A. Bottino, P. Angelino, S. J. Allfrey et al., in Theory of Fusion Plasmas, Int. Workshop (Editrice Compositori, Societá italiana di Fisica, Bologna, 2004), p. 75.

${ }^{9}$ T. Tran, K. Appert, M. Fivaz, G. Jost, J. Vaclavik, and L. Villard, in Theory of Fusion Plasmas, Int. Workshop (Editrice Compositori, Societá italiana di Fisica, Bologna, 1999), p. 45.

${ }^{10}$ P. Angelino, A. Bottino, R. Hatzky et al., Phys. Plasmas 13, 052304 (2006).

${ }^{11}$ J. A. Krommes, Phys. Plasmas 6, 1477 (1999).

${ }^{12}$ R. Hatzky, T. Tran, A. Könies, R. Kleiber, and S. Allfrey, Phys. Plasmas 9, 898 (2002).

${ }^{13}$ T. Hahm, Phys. Fluids 31, 2670 (1988).

${ }^{14}$ A. M. Dimits, G. Bateman, M. A. Beer et al., Phys. Plasmas 7, 969 (2000).

${ }^{15}$ A. Bottino, A. G. Peeters, R. Hatzky et al., Europhysics Conference Abstract, 33rd European Physical Society Conference on Plasma Physics, Rome, June, 2006, Paper, O3.001; http://eps2006.frascati.enea.it/invited/ orals.htm

${ }^{16}$ A. Aydemir, Phys. Plasmas 1, 822 (1994). 